\title{
A PFC Voltage Regulator With Low Input Current Distortion Derived From a Rectifierless Topology
}

\author{
Joe C. P. Liu, Member, IEEE, Chi K. Tse, Fellow, IEEE, N. K. Poon, Member, IEEE, \\ Bryan M. H. Pong, Senior Member, IEEE, and Y. M. Lai, Member, IEEE
}

\begin{abstract}
This paper presents an ac-de converter topology for realization of power factor correction (PFC) voltage regulators for applications where the mains frequency is high and a low input current harmonic is required, e.g., in aircraft power systems. The proposed converter represents a minimal configuration consisting of two basic converters, which can be systematically derived from a previously proposed general synthesis procedure for rectifierless ac-dc converters. The proposed PFC converter has incorporated a control method which drastically reduces the circulating power and hence raises the efficiency to a level comparable to existing PFC converters. The proposed PFC converter can completely eliminate any crossover distortion, which can be significant for supply systems having a high mains frequency. In addition, the proposed converter allows bidirectional energy flow ensuring all inductors work in continuous conduction mode hence eliminating the distortion due to the abrupt change of dynamic response when the operating mode changes. Analysis and design of the power and control circuits will be given and discussed. An experimental system will be presented for verification purposes.
\end{abstract}

Index Terms-AC-DC converter, airborne application, low distortion, power factor correction (PFC), rectifierless converter.

\section{INTRODUCTION}

$\mathbf{T}$ HE AIM of power-factor-correction (PFC) converters is to reduce the input current harmonics and provide high power factor to the ac mains supply. A bridge rectifier followed by a boost converter operating at critical conduction mode or in continuous conduction mode under average current mode control is being widely used for PFC applications because of its simplicity [1], [2]. Researchers have revealed two major distortions in boost type PFC configurations [3], [4].

1) It has been shown by Sun [3] that the use of the average current mode control method and a filtering capacitor placed after the input bridge rectifier for eliminating high-frequency ripple current will introduce a leading phase to the input current relative to the input mains. Near the zero crossing point of the input ac voltage, the input

Manuscript received January 14, 2005; revised August 16, 2005. This paper was presented in part at the IEEE Power Electronics Specialists Conference, Aachen, Germany, 2004. This work was supported by the Hong Kong Research Grants Council under Research Grant PolyU 5237/04E. Recommended by Associate Editor J. Cobos.

J. C. P. Liu and N. K. Poon are with Powerelab Limited, Department of Electrical and Electronic Engineering, University of Hong Kong, Hong Kong, China (e-mail: cpliu@eee.hku.hk).

C. K. Tse and Y. M. Lai are with the Department of Electronic and Information Engineering, Hong Kong Polytechnic University, Hong Kong, China (e-mail: encktse@polyu.edu.hk).

B. M. H. Pong is with the Department of Electrical and Electronic Engineering, University of Hong Kong, Hong Kong, China.

Digital Object Identifier 10.1109/TPEL.2006.876887

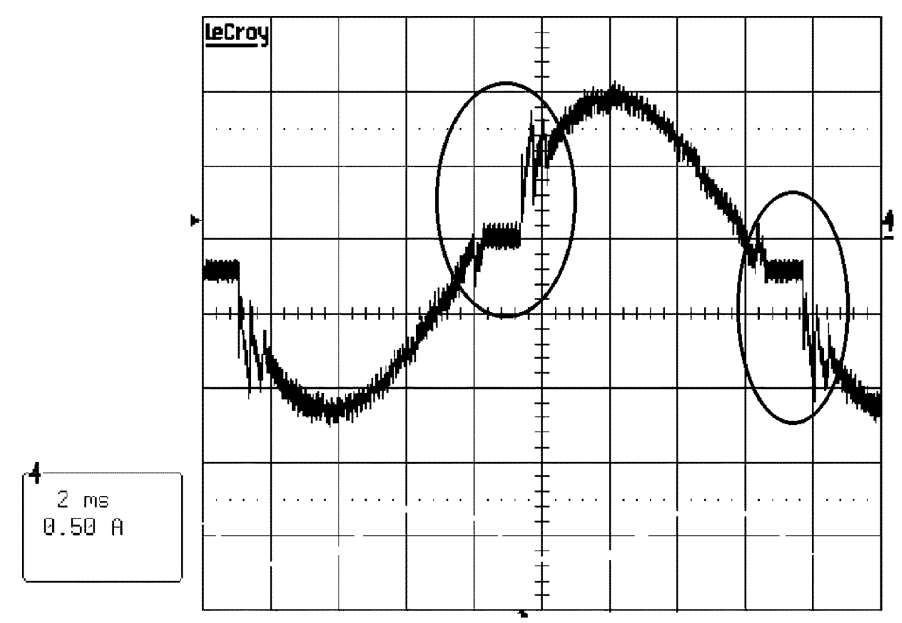

Fig. 1. Crossover distortion.

current reverses in advance of the voltage due to the phase lead, and the input bridge rectifier will be reverse biased and cease to conduct current. This causes a step change in the input current and gives rise to crossover distortion. The significance of this distortion increases with the mains frequency and can be very serious when the power system operates at $400 \mathrm{~Hz}$ or above in airborne applications. Fig. 1 shows the crossover distortion of input current of a PFC caused by the phase lead of input current to the mains voltage and the input bridge rectifier.

2) Another distortion is caused by the change of operating mode of the boost inductor when the load and/or input voltage is changed. The dynamics of the converter changes abruptly, causing distortion to the input current. Although critical conduction mode operation can alleviate the problem, it is undesirable for high power applications since the high ripple current flow in the inductor and switches increases the conduction loss. Moreover, the problem can be reduced by adding a special correction factor to the input current sampling circuit [4], at the expense of an increased complexity of the control circuits. Fig. 2 shows the distortion at the change of operating mode.

In this paper, we propose a PFC converter topology that can eradicate the distortions described above. This topology is derived from the general synthesis procedure for rectifierless ac-dc conversion topologies presented in Liu et al. [5]. It represents a minimal configuration consisting of only two basic converters that can accomplish the required objectives of PFC and voltage regulation. The basic idea is to use two 


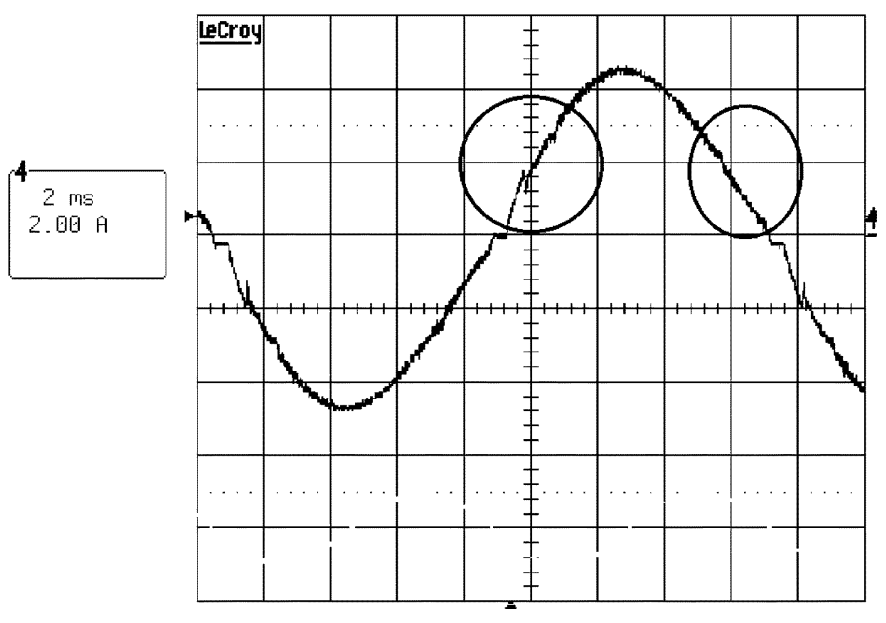

Fig. 2. Distortion due to mode change.

dc-dc converters to provide a dc stacking voltage to the ac input voltage, thereby preventing polarity change of the input voltage and hence eliminating the need for input rectifiers. The specific converter configuration chosen for this application is shown in Fig. 3(a). Also, the specific choice of constituent dc-dc converters, as shown in Fig. 3(b), has been found to give lowest voltage stress on the switches. Moreover, it has been revealed [5] that this rectifierless topology suffers from circulating power loss, which becomes significant when the stacking voltage is large. Thus, in this paper, instead of using a constant dc stacking voltage, we introduce a variable stacking voltage, resulting in significant reduction of the circulating power and loss.

A remark worth mentioning, before we describe details of the circuit, is that the circuit looks a prima facie kind of fourquadrant converter. However, we demonstrate in this paper how such a circuit can be systematically decomposed into two basic $\mathrm{dc}-\mathrm{dc}$ converters, thus allowing suitable control strategies to be more readily developed to satisfy any desired operation.

This paper is organized as follows. In Section II, we will review the operation of the proposed circuit, and explain how circulating power loss can be reduced in this circuit. In Section III we will present experimental results verifying the operation of the circuit and its ability of eliminating distortion at high line frequency $(400 \mathrm{~Hz})$. Finally, Section IV gives the conclusion.

\section{OPERATION ANALYSIS}

The power conversion stage consists of two dc-dc bidirectional converters, as shown in Fig. 3(b). DC-DC converters 1 and 2 are boost and buck converter according to the power flow directions shown by the solid arrows. To realize the input rectifierless ac-dc conversion, dc-dc converter 2 should provide a stacking voltage $\xi(t)$ to the mains Vi such that the input voltage of dc-dc converter $1, V_{1}$, is always positive regardless of $V_{i}$. To satisfy this condition, the output voltage $U$ and $\xi(t)$ must satisfy

$$
U>D_{2}(t) U=\xi(t)>E \sin 2 \pi f_{e} t \quad \text { for all } t
$$

where all symbols are defined in Fig. 3.

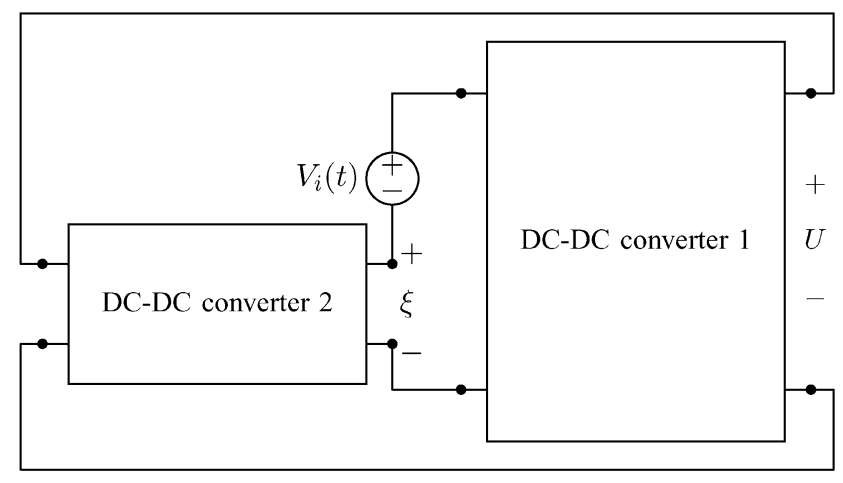

(a)

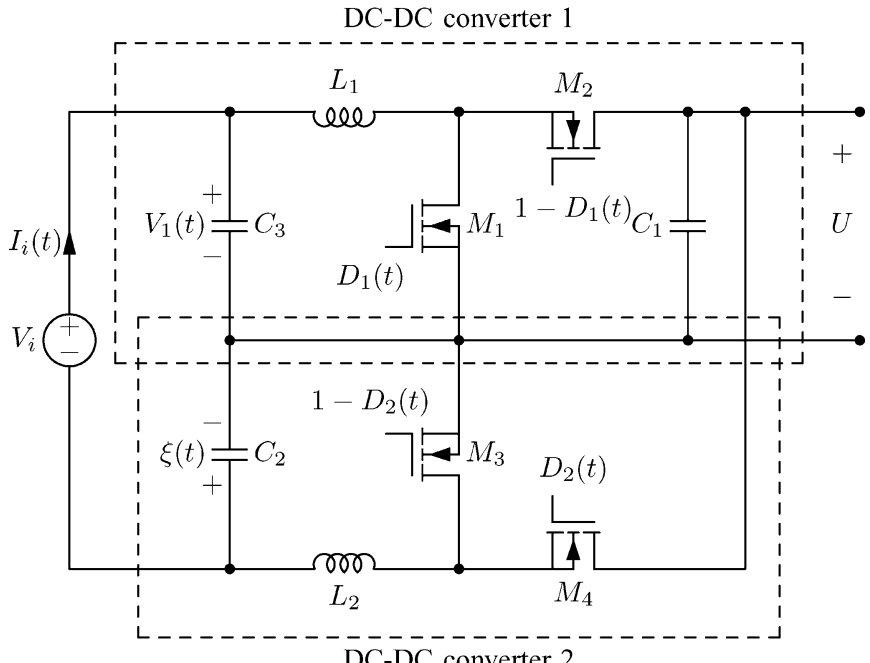

(b)

Fig. 3. Rectifierless ac-dc converter circuit: (a) general configuration [5] and (b) specific circuit choice with the boost and buck converters realizing the two constituent dc-dc converters.

This implies that $U$ must be greater than the amplitude of the input ac voltage $E$ and the overall conversion is a step-up operation. Since the conversion ratio of dc-dc converter 1 is equal to $1 /\left(1-D_{1}(t)\right)$, the relationship between $U, V_{i}, D_{1}(t)$, and $D_{2}(t)$ is given by

$$
U=\frac{V_{i}}{1-D_{1}(t)-D_{2}(t)}
$$

\section{A. Reduction of Circulating Power}

The topology used for PFC converter implementation has a drawback in conversion efficiency due to circulation power. It has been shown in [5] that the circulating power is equal to $4 \xi / \pi E$ times the output power $P_{o}$ when stacking voltage $\xi$ is kept constant. This means that the minimum circulating power flows around dc-dc converters 1 and 2 is $4 P_{o} / \pi$ since $\xi$ must be greater than $E$. To reduce the circulating power, we propose to add a time varying component, which is in anti-phase to $V_{i}$, to the stacking voltage in order to provide "just enough"stackup dc voltage during the negative cycle. It can be done by equating the duty cycle of $M_{1}$ and $M_{4}$, i.e., $D_{2}(t)=D_{1}(t)=D(t)$. Under this condition, the stacking voltage $\xi(t)$ as well as the 
output voltage $U$ and the input voltage to dc-dc converter 1 can be derived as follows.

$$
\begin{aligned}
U & =\frac{V_{i}}{1-2 D(t)} \text { or } D(t)=\frac{1}{2}\left(1-\frac{V_{i}}{U}\right) \\
\xi(t) & =D(t) U=\frac{1}{2}\left(U-V_{i}\right) \\
V_{1}(t) & =\xi(t)+V_{i}=\frac{1}{2}\left(U+V_{i}\right) .
\end{aligned}
$$

We can see that $\xi(t)$ contains a time varying component $V_{i} / 2$, which is in antiphase to $V_{i}$. In addition, (5) shows that $V_{1}$ is always positive with $U>E$ and satisfies the basic requirement.

To derive the circulating power when the converter operates for PFC, we can assume that the input current $I_{i}$ is controlled to follow the shape of the mains $V_{i}$ with amplitude equal to $2 P_{o} / E \eta_{\text {overall }}$, where $\eta_{\text {overall }}$ is the overall efficiency of the converter, i.e.,

$$
I_{i}=\frac{2 P_{o}}{E \eta_{\text {overall }}} \sin 2 \pi f_{e} t .
$$

Then, the circulating power $P_{\text {circulate }}$ is given by [5]

$$
\begin{aligned}
P_{\text {circulate }} & =2 f_{e} \int_{0}^{1 / 2 f_{e}}\left(V_{i}+\xi(t)\right) I_{i} \cdot d t-P_{o} \\
& =\left(\frac{2 U}{\pi E \eta_{\text {overall }}}-\frac{1}{2}\right) P_{o} .
\end{aligned}
$$

It can be seen that the minimum circulation power $(2 / \pi-$ $1 / 2) P_{o}$ can be achieved when $U$ is made close to $E$. The circulating power is reduced by a factor of 9.32 when compared with $4 P_{o} / \pi$.

Obviously, if $\eta$ is the efficiency of each constituent converter, the total loss due to the circulating power is

$$
\operatorname{loss}_{\text {circulate }}=2 P_{\text {circulate }}\left(\frac{1}{\eta}-1\right) .
$$

Also, as power goes directly to the output through dc-dc converter 1 , the loss is

$$
\operatorname{loss}_{\text {direct }}=P_{o}\left(\frac{1}{\eta}-1\right) \text {. }
$$

Hence, the overall efficiency is

$$
\begin{aligned}
\eta_{\text {overall }} & =\frac{P_{o}}{P_{o}+\operatorname{loss}_{\text {circulate }}+\operatorname{loss}_{\text {direct }}} \\
& =\frac{P_{o}}{P_{o}+P_{o}\left(\frac{1}{\eta}-1\right)\left(\frac{4 U}{\pi E \eta_{\text {overall }}}\right)} \\
& =1-\frac{4 U}{\pi E}\left(\frac{1}{\eta}-1\right) .
\end{aligned}
$$

Alternatively, we can write

$$
\eta=\frac{1}{1+\frac{\pi E}{4 U}\left(1-\eta_{\text {overall }}\right)}
$$

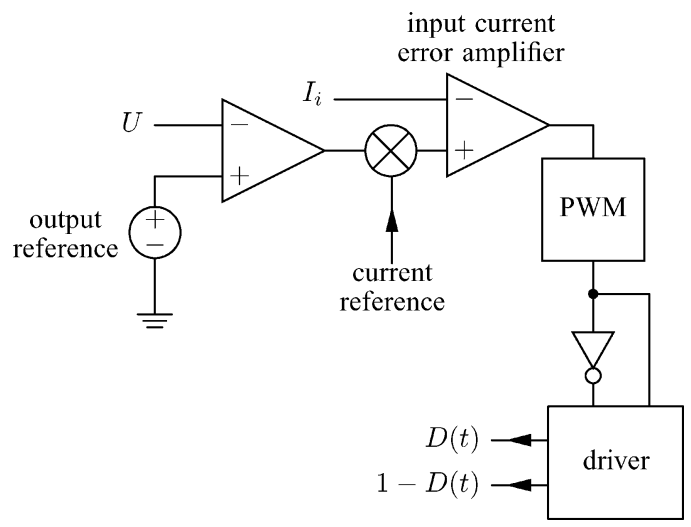

Fig. 4. Simplified schematic of the control circuit for power factor correction. Current reference is derived from $V_{i}$. Output reference is derived from the amplitude of $V_{i}$.

which can be used to estimate the constituent converter efficiency from the measured overall efficiency.

Remarks: From the foregoing discussion, the two converters seem to be processing power redundantly, doubling the overall converter size. However, using the proposed control of the circulating power, the net increase in the power processed by each converter is not significant. For instance, under the conditions of an anti-phase stacking voltage and the afore-described controlled output voltage, the maximum circulating power is about $13.67 \%$ of $P_{o}$. During the positive half cycle of the input voltage, converter 1 processes an average power of $1.1367 P_{o}$, while converter 2 processes an average power of $0.1367 P_{o}$. By symmetry, during the negative half cycle, converter 2 processes an average power of $1.1367 P_{o}$ while converter 1 processes an average power of $0.1367 P_{o}$ Therefore, each converter processes $0.6367 P_{o}$, and the whole converter thus processes an average power of $1.2733 P_{o}$. The overall increase of the converter size is not doubled, but only about $27.33 \%$.

\section{B. Control Circuit Realization for PFC}

The simplified control circuit shown in Fig. 4 is used for the PFC control. The input current error amplifier forces the input current to follow the reference derived from $V_{i}$. The reference voltage for regulating the output voltage $U$ is made proportional to the input voltage amplitude. This makes $U$ decrease with $E$ and reduces the ratio $U / E$ at low ac voltage input which helps to reduce circulation power derived in (7). The theoretical basis of the control possibility has been discussed in [2],[6].

\section{EXPERIMENTAL RESULTS}

A PFC converter using the proposed topology has been built for verification. The operating switching frequency is $50 \mathrm{kHz}$. The output voltage was made to vary with the input mains amplitude as described in the previous section. The current sensing has been implemented indirectly as shown in Fig. 5. Since the input current is proportional to the differential voltage across the sensing resistors $R_{\mathrm{s} 1}$ and $R_{\mathrm{s} 2}$, it can be obtained using a differential amplifier as shown in Fig. 5. All MOSFETs are IRFB18N50. Also, the input filter takes the form of a standard 
TABLE I

EXPERIMENTAL RESULTS

\begin{tabular}{cllll}
\hline Test & Input Voltage & Output Voltage $U$ & Output Power $P_{o}$ & Measured Efficiency $\eta_{\text {overall }}$ \\
\hline \hline 1 & $90 \mathrm{~V}(\mathrm{rms})$ & $174.4 \mathrm{~V}$ & $100 \mathrm{~W}$ & $\mathbf{9 3 . 8} \%$ (with circulating power control) \\
2 & $90 \mathrm{~V}(\mathrm{rms})$ & $382.6 \mathrm{~V}$ & $100 \mathrm{~W}$ & $87.3 \%$ (without circulating power control) \\
3 & $230 \mathrm{~V} \mathrm{(rms)}$ & $380.2 \mathrm{~V}$ & $100 \mathrm{~W}$ & $\mathbf{9 4 . 5} \%$ (with circulating power control) \\
\hline
\end{tabular}

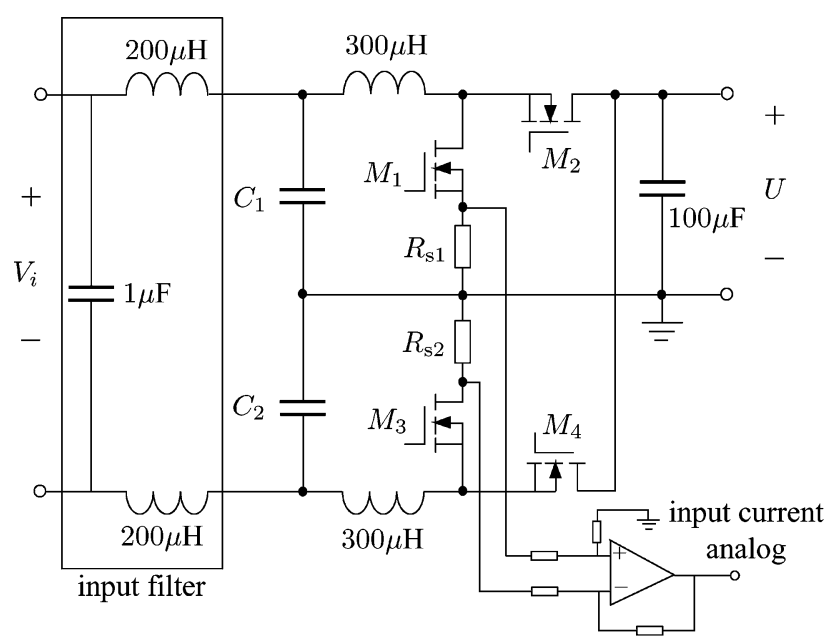

Fig. 5. Experimental circuit showing input current sensor and input filter. The current sensing circuit makes use of the property that the input current is proportional to the differential voltage across $R_{\mathrm{s} 1}$ and $R_{\mathrm{s} 2}$.

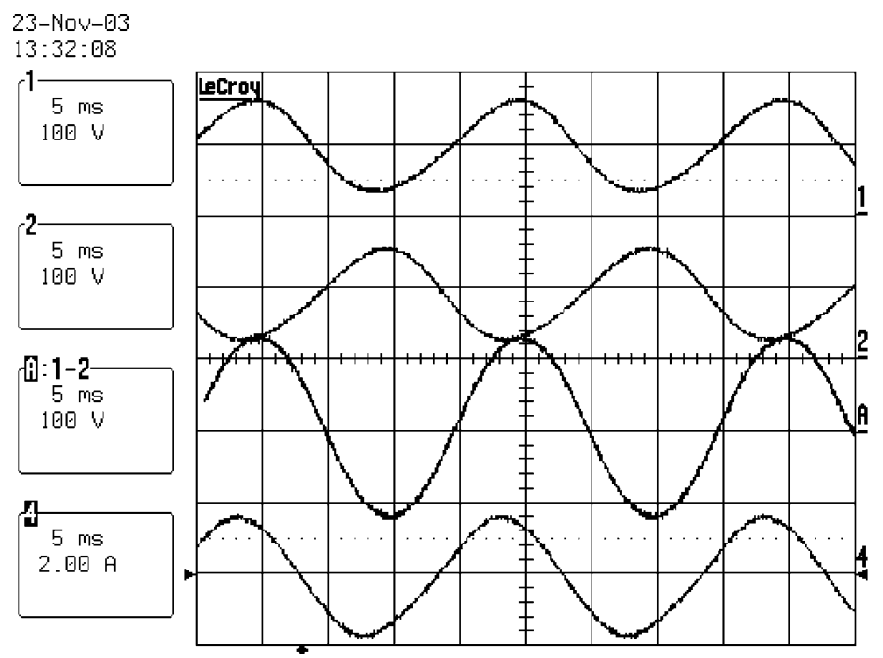

Fig. 6. Waveforms of $V_{1}(\mathrm{Ch} 1), \xi(t)(\mathrm{Ch} 2), V_{i}(\mathrm{ChA})$, and $I_{i}(\mathrm{Ch} 4)$, for input voltage $90 \mathrm{~V}(\mathrm{rms})$ and line frequency $50 \mathrm{~Hz}$.

differential-mode filter, which consists of two $200 \mu \mathrm{H}$ inductors and a $1 \mu \mathrm{F}$ capacitor.

Some test results are shown in Table I. From the results of Test 1 and using (11), we can estimate the constituent converter efficiency $\eta$, i.e., $\eta=96.6 \%$. We can check consistency with the results of Test 2. Assuming $\eta=96.6 \%$ for Test 2 (without circulating power control), we get the overall efficiency as $86.5 \%$, which agrees with the measured one. Test 3 repeats Test 2 with a different set of input and output voltages.

Fig. 6 shows the operating waveforms of $V_{1}, \xi(t), V_{i}$, and $I_{i}$. We can see that $\xi(t)$ is in anti-phase to $V_{i}$ and its magnitude

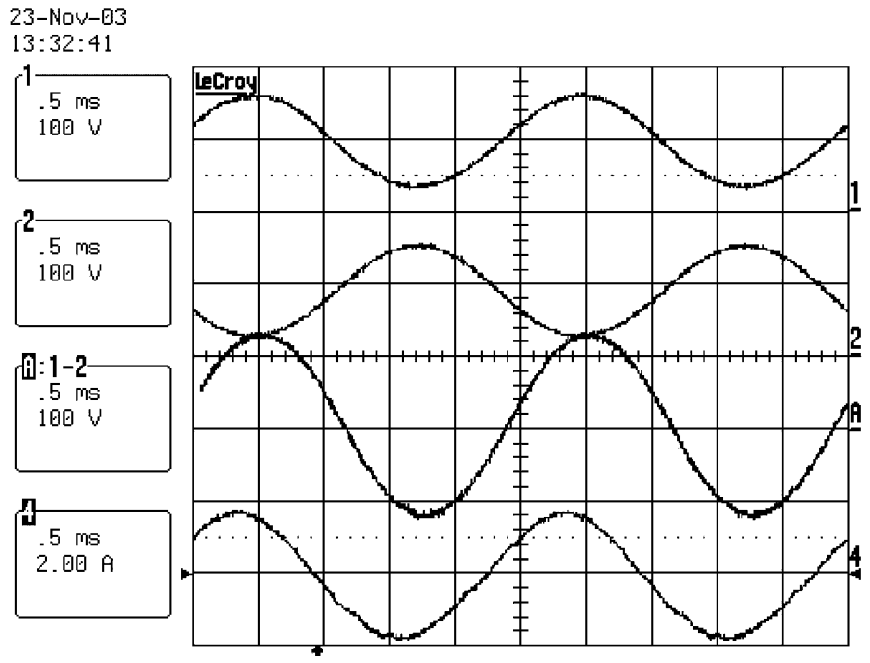

Fig. 7. $\left\{\right.$ Waveforms of $V_{1}(\mathrm{Ch} 1), \xi(t)(\mathrm{Ch} 2), V_{i}(\mathrm{ChA})$, and $I_{i}(\mathrm{Ch} 4)$, for input voltage $90 \mathrm{~V}(\mathrm{rms})$ and line frequency $400 \mathrm{~Hz}$.

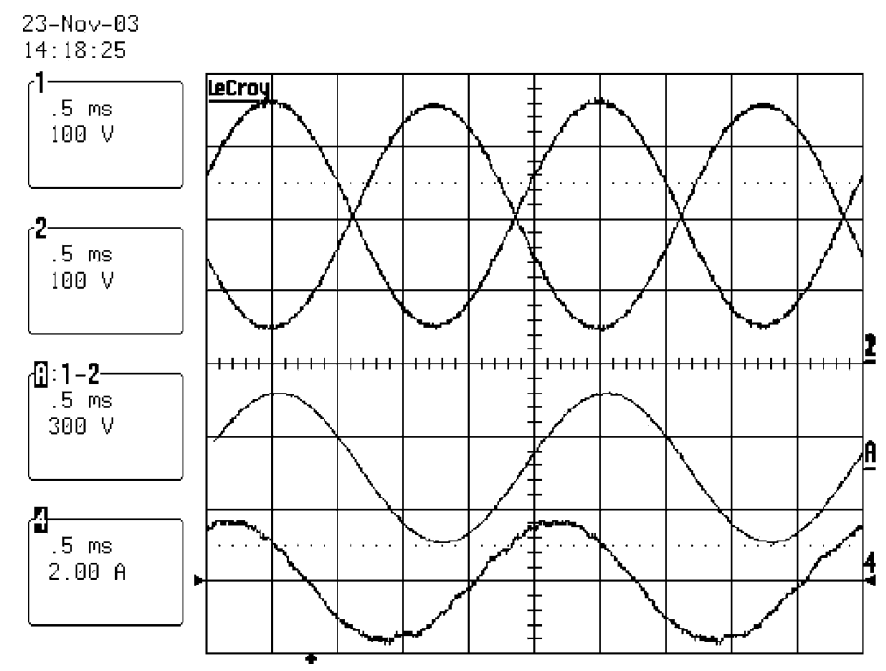

Fig. 8. Waveforms of $V_{1}(\mathrm{Ch} 1), \xi(t)(\mathrm{Ch} 2), V_{i}(\mathrm{ChA})$ and $I_{i}(\mathrm{Ch} 4)$, for input voltage $230 \mathrm{~V}(\mathrm{rms})$ and line frequency $400 \mathrm{~Hz}$.

reduces when $V_{i}$ is positive in order to reduce the circulation power.

Figs. 7 and 8 show the operating waveforms at $400-\mathrm{Hz}$ mains frequency and no significant distortion has been found. Fig. 9 is the blow-up waveforms of $V_{i}$ and $I_{i}$, and no crossover distortion has been observed even when a noticeable phase lead of the input current due to the input filter is present at $230-\mathrm{V}, 400-\mathrm{Hz}$ input.

Fig. 10 show the operating duty cycle at different values $(0 \mathrm{~V}$ and $300 \mathrm{~V}$ ) of $V_{i}$. The calculated (using (3)) and the measured duty cycles are in perfect agreement. At $V_{i}=0$ the calculated 


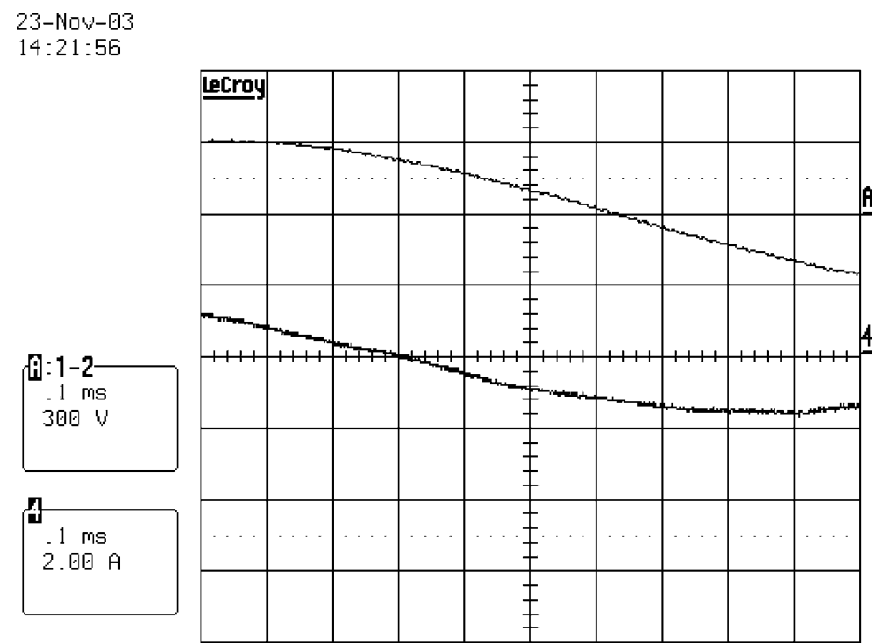

Fig. 9. Close-up view of waveforms of $V_{i}(\mathrm{ChA})$ and $I_{i}$ (Ch4), for input voltage $230 \mathrm{~V}(\mathrm{rms})$ and line frequency $400 \mathrm{~Hz}$.

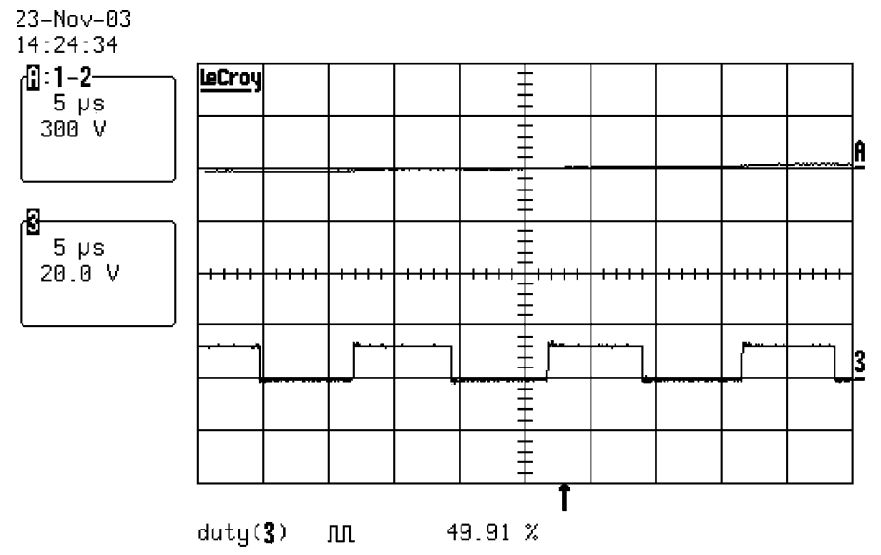

(a)

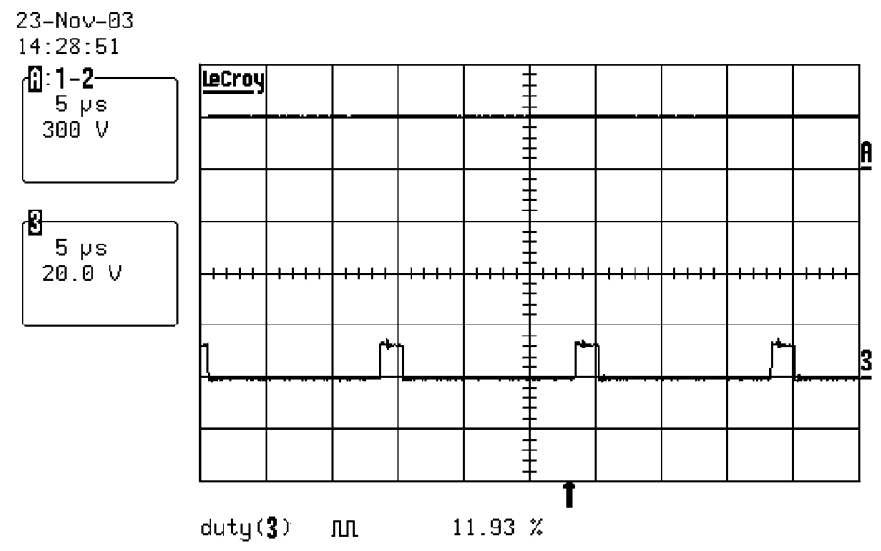

(b)

Fig. 10. Waveforms of $V_{i}(\mathrm{ChA})$ and $D(t)(\mathrm{Ch} 3)$ at (a) zero crossing point and (b) input voltage $300 \mathrm{~V}$.

and measured duty cycle are 0.5 and 0.499 while at $V_{i}=300 \mathrm{~V}$ the calculated and measured duty cycle are 0.105 and 0.119 , respectively.

\section{CONCLUSION}

An input rectifierless PFC converter with low input current distortion has been proposed and verified experimentally. It eradicates the crossover distortion due to the input rectifier and the phase lead of input current introduced by the filter and control circuits in conventional PFC converters, which is a significant problem at high mains frequencies. No distortion due to mode changing has been found since the converter allows bidirectional energy flow and all inductors work at continuous conduction mode at all times. An important design feature of this converter is that a control circuit has been designed to reduce the circulation power and provide higher conversion efficiency. This makes the proposed PFC converter suitable for airborne power systems requiring low input current harmonics with a relatively high mains frequency. Possible future work may proceed along the idea of rectifierless configurations [7] as well as other practical design tradeoff considerations [8]. Finally, it should be pointed out that the two-converter configuration described in this paper represents a minimal converter configuration that can be used to emulate any input impedance, not limited to pure resistance [9].

\section{REFERENCES}

[1] R. Redl, "Power-factor-correction in single-phase switching-mode power supplies," Int. J. Electron., vol. 77, no. 5, pp. 555-582, May 1994.

[2] C. K. Tse, "Circuit thoery of power factor correction in switchingconverters," Int. J. Circuit Theory Appl., vol. 31, no. 2, pp. 157-198, Mar. 2003.

[3] J. Sun, "Demystifying zero-crossing distortion in single-phase PFC converters," in Proc. IEEE Power Electron. Spec. Conf., Jun. 2002, pp. $1109-1114$.

[4] K. de Gusseme, D. M. V. de Sype, A. P. van den Bossche, and J. A. Melkebeek, "Sample correction for digitally controlled boost PFC converters operating in both CCM and DCM," in Proc. IEEE Appl. Power Electron. Conf. Expo, Feb. 2003, pp. 389-395.

[5] C. P. Liu, C. K. Tse, N. K. Poon, B. M. H. Pong, and Y. M. Lai, "Synthesis of input-rectifierless AC-DC converters," IEEE Trans. Power Electron., vol. 19, no. 1, pp. 176-182, Jan. 2004.

[6] C. K. Tse and M. H. L. Chow, "Theoretical study of switching converters with power factor correction and output regulation," IEEE Trans. Circuits Syst., vol. 47, no. 7, pp. 1047-55, Jul. 2000.

[7] A. Ikriannikov and S. Ćuk, "Direct ac-dc conversion without input rectification," in Proc. IEEE Power Electron. Spec. Conf., Jun. 1999, pp. $181-186$.

[8] C. Zhou and M. Jovanović, "Design trade-offs in continuous currentmode controlled boost power-factor correction circuits," in Proc. High Freq. Power Conv. Conf., Apr. 1992, pp. 209-220.

[9] J. C. P. Liu, C. K. Tse, N. K. Poon, M. H. Pong, and Y. M. Lai, "General impedance synthesizer using minimal configuration of switching converters," in Proc. Eur. Conf. Circuit Theory Design, Cork, Ireland, Aug. 2005, pp. 1-4.

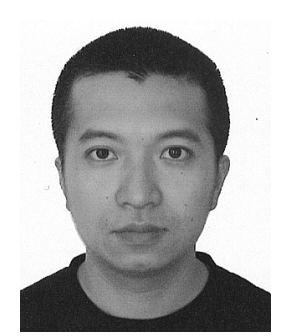

Joe C. P. Liu (M'99) was born in Hong Kong in 1970. He received the B.Sc. degree in electrical and electronic engineering from the University of Hong Kong, Hong Kong, China, in 1993 and is currently pursuing the Ph.D. degree at Hong Kong Polytechnic University.

From 1993 to 1999, he held several industrial positions in switching power supply design. In 1999, he joined the Power Electronics Laboratory, University of Hong Kong, as a Principal Research Engineer. Presently, he is a Principal Research Engineer with Powerelab Limited, Hong Kong. His research interest includes switching converter topologies, synchronous rectifiers, soft switching, and EMI modeling. 


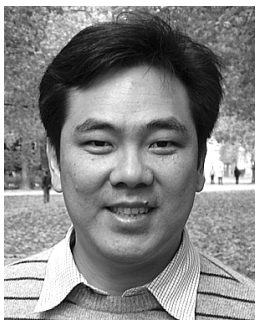

Chi K. Tse (M'90-SM'97-F'06) received the B.Eng. degree (with first class honors) in electrical engineering and the Ph.D. degree from the University of Melbourne, Australia, in 1987 and 1991, respectively.

$\mathrm{He}$ is presently Chair Professor and Head of Department of Electronic and Information Engineering, Hong Kong Polytechnic University, Hong Kong, China. Since 2002, he has been Guest Professor with the Southwest China Normal University, Chongqing, China. He is the author of Linear Circuit Analysis (London, U.K.: Addison-Wesley 1998) and Complex Behavior of Switching Power Converters (Boca Raton, FL: CRC Press, 2003), coauthor of Chaos-Based Digital Communication Systems (Heidelberg, Germany: Springer-Verlag, 2003) and Chaotic Signal Reconstruction with Applications to Chaos-Based Communications (Beijing, China: TUP, 2005). He is co-holder of a U.S. patent and two pending patents. Presently, he serves as an Associate Editor for the International Journal of Systems Science. His research interests include power electronics and nonlinear systems.

Dr. Tse received the L.R. East Prize from the Institution of Engineers, Australia, in 1987, the IEEE TRANSACTIONS ON POWER ElECTRONICS Prize Paper Award in 2001, the International Journal of Circuit Theory and Applications Best Paper Award in 2003, the Hong Kong Polytechnic University President's Award for Achievement in Research twice, the Faculty's Best Researcher Award, and the Research Grant Achievement Award. From 1999 to 2001, he was an Associate Editor for the IEEE TRANSACTIONS ON CIRCUITS AND SYSTEMS: PART I-FUNDAMENTAL THEORY AND APPLICATIONS and since 1999 has been an Associate Editor for the IEEE TRANSACTIONS ON POWER ELECTRONICS. He was an IEEE Distinguished Lecturer in 2005.

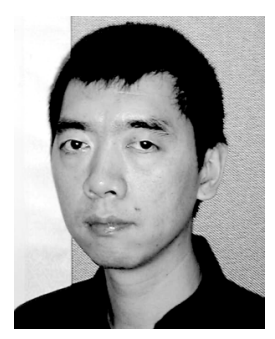

N. K. Poon (M'95) received the B.Eng. degree (with honors) in electronic engineering from the City University of Hong Kong, Hong Kong, China, in 1995 and the Ph.D. degree from the Hong Kong Polytechnic University, Hong Kong, in 2003.

After graduation, he worked with Artesyn Technologies (Asia Pacific) Limited and Power Electronics Laboratory, University of Hong Kong. $\mathrm{He}$ is presently with Powerelab Limited, Hong Kong, where he leads a research team working in computer-aided design, soft switching techniques,
EMI modeling, PFC topologies, synchronous rectification, converter modeling, PWM inverters, and fast transient regulators.

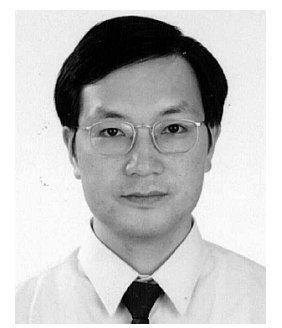

Bryan M. H. Pong (M'84-SM'96) was born in Hong Kong. He received the B.Sc. degree in electronic and electrical engineering from the University of Birmingham, Birmingham, U.K., in 1983 and the Ph.D. degree in power electronics from Cambridge University, Cambridge, U.K., in 1987.

After graduation, he became a Senior Design Engineer and then a Chief Design Engineer at National Semiconductor Hong Kong, China, where he was involved in electronic product design. Afterwards, he joined ASTEC International, Hong Kong, first as a Principal Engineer and then a Division Engineering Manager. He is now an Associate Professor with the University of Hong Kong, where he is in charge of the Power Electronics Laboratory and leads a team to carry out research in switching power supplies. He co-holds a number of U.S. patents. His research interests include synchronous rectification, EMI issues, power factor correction, magnetic component design, soft switching and digital control.

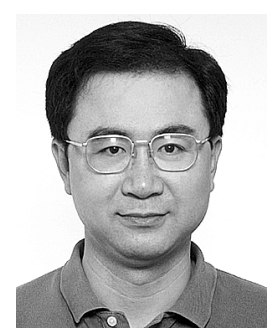

Y. M. Lai (M'92) received the B.Eng. (with honors) degree in electrical engineering from the University of Western Australia, Perth, Australia, in 1983, the M.Eng.Sc. degree in electrical engineering from the University of Sydney, Sydney, Australia, in 1986, and the Ph.D. degree from Brunel University, West London, U.K., in 1997.

$\mathrm{He}$ is currently an Assistant Professor in the Department of Electronic and Information Engineering, Hong Kong Polytechnic University, Hong Kong, China. Prior to joining the University, he held technical positions with National Semiconductor, Derek Philips Associates, and Siliconix Hong Kong Limited. His research interests include nonlinear dynamics and sliding mode control of power converters. 